Published online 2017 April 13.

Abstract

\title{
Assessment of Correlation of Left Ventricular Diastolic Functional Indices Between Gated Myocardial Perfusion SPECT and Echocardiography
}

\author{
Lida Peivandi, ${ }^{1,}$ and Vahid Reza Dabbagh Kakhki ${ }^{2}$ \\ 'Zahedan University of Medical Sciences, Zahedan, Iran \\ ${ }^{2}$ Mashhad University of Medical Sciences, Mashhad, Iran \\ "Corresponding author: Lida Peivandi, Zahedan University of Medical Sciences, Zahedan, Iran. E-mail: lida.peivandi@gmail.com
}

Received 2016 December 21; Accepted 2017 February 08.

\begin{abstract}
Background: Left ventricular diastolic function assumes as a major morbidity and mortality risk factor in cardio-vascular diseases especially in patients with heart failure with preserved ejection fraction. In myocardial gated perfusion SPECT, both the perfusion and the LV function, specialy systolic ones, are evaluated. Though diastolic indices, assessed by SPECT, are often overlooked in reports and they were not compared with the known methods for assessment of LV diastolic function, best of them is echocardiography (Doppler and 2D). The aim of this study was to assess the correlation of LV diastolic indices with echocardiography.

Methods: Forty-nine consecutive patients referred to Ghaem hospital, Nuclear medicine ward, between 2013 to 2016, were performing both SPECT and echocardiography. Diastolic LV indices in SPECT were calculated with QGS software and all DATA were recorded in SPSS for statistical analysis by performing correlation studies. P value less than 0.05 is defined as significant level.

Results: The population study consisted of 19 male and 30 female with the average age of 58.2 years.There is a mild negative correlation between PFR, MFR/3 and PFR/2 with LA volume, LA diameter and LA index. There is a mild negative correlation between MFR/3 and Posterior wall thickness and also a mild positive correlation between PFR/2 and systolic LV diameter. There is no significant correlation between SPECT diastolic indices with other variables in echocardiography.

Conclusions: In this study there is no correlation between the main LV diastolic indices derived by SPECT and echocardiography, may be due to the main changes in the last version of guidline for assessment of LV diastolic function by echocardiography.

Keywords: Diastolic Dysfunction, Echocardiography, SPECT, Diastolic Indices, Heart Failure
\end{abstract}

This is an abstract presented in the 33rd Iranian congress of radiology (ICR) and the 15th congress of Iranian radiographic science association (IRSA). 